\title{
Convenient synthesis of 3,5,7-trimethyl-1-azonia-adamantanes
}

\author{
Hiroshi Izumi* and Shigeru Futamura \\ National Institute for Resources and Environment, 16-3 Onogawa, \\ Tsukuba, Ibaraki 305-8569, Japan \\ E-mail: izumi@nire.go.jp
}

(received 25 Sep 99; accepted 13 Feb 00; published on the web 21 Feb 00)

\begin{abstract}
A convenient synthesis of 3,5,7-trimethyl-1-azonia-adamantanes (2) is described. The esterification of cis,cis-1,3,5-tris(hydroxymethyl)-1,3,5-trimethylcyclohexane (3) with trifluoromethanesulfonic anhydride, followed by the reaction with primary amines, yields azonia-adamantanes 2. On the other hand, the esterification of triol 3 with TFAA also affords cis,cis-1,3,5-tris[(trifluoroacetoxy)methyl]-1,3,5-trimethylcyclohexane (5). However, the reaction of triester 5 with 2-(2-aminoethyl)pyridine does not give the corresponding azoniaadamantane, but 2,2,2-trifluoro- $\mathrm{N}$-[2-(pyridin-2-yl)ethyl]-acetamide (6). The $\beta$-methylene protons in azonia-adamantane $2 \mathrm{a}[\mathrm{R}=2$-(pyridin-2-yl)ethyl $]$ are very active. Both acids and bases react with $2 \mathrm{a}$ to yield 2 -vinylpyridine. The elimination reaction of $2 \mathrm{a}$ with lithium methoxide is available for the synthesis of 3,5,7-trimethyl-1-aza-adamantane (1).
\end{abstract}

Keywords: Azonia-adamantanes, lithium methoxide, vinylpyridine

\section{Introduction}

3,5,7-Trimethyl-1-aza-adamantane structures (1) have been applied as highly twisted amides, ${ }^{1}$ self-organization systems, ${ }^{2}$ and rigid models. ${ }^{3}$ These structures have been also attracting considerable interest because of their pharmacological activity. ${ }^{4}$ In contrast, there have been few studies on 3,5,7-trimethyl-1-azonia-adamantanes 2, and many synthetic steps have been needed. ${ }^{5}$ In this paper, we report a convenient synthetic method for 3,5,7-trimethyl-1-azonia-adamantanes 2. We also present an elimination reaction of $2 \mathrm{a}[\mathrm{R}=2$-(pyridin-2-yl)ethyl $]$ to give azoniaadamantane $2 \mathrm{c}(\mathrm{R}=\mathrm{H})$ and 2-vinylpyridine. 


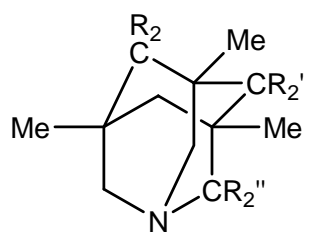

1

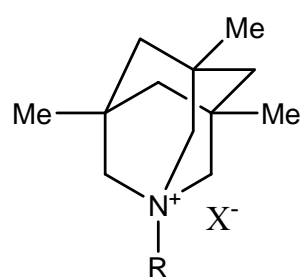

2

\section{Results and Discussion}

As shown in Scheme 1, the esterification of cis,cis-1,3,5-tris(hydroxymethyl)-1,3,5trimethylcyclohexane (3) ${ }^{6}$ with trifluoromethanesulfonic anhydride (4 equiv), ${ }^{7}$ followed by reactions with primary amines (4 equiv), gave 3,5,7-trimethyl-1-azonia-adamantanes 2 [2a, $\mathrm{R}=$ 2-(pyridin-2-yl)ethyl, 23\%; 2b, $\mathrm{R}=4$-methoxybenzyl, 31\%]. The ${ }^{1} \mathrm{H}$ NMR, ${ }^{13} \mathrm{C}$ NMR (DEPT),${ }^{1} \mathrm{H}^{-13} \mathrm{C}$ COSY spectra supported the structure of 2 . Mayer and co-workers indicated that triester 4 ( $\mathrm{X}=$ trifluoromethylsulfonyloxy) is easily obtained by the reaction of triol 3 with trifluoromethanesulfonic anhydride and is available for the trisubstituted tripodal ligand. ${ }^{7}$ In this case, however, the corresponding triamines could not be obtained. We have already shown that the steric repulsion of the ipso methyl groups in the cyclohexane ring causes the unexpected reactions ${ }^{8}$ and stabilizes the molecular structures. ${ }^{9}$ This finding suggests that the proximity effect works efficiently in the synthesis of azonia-adamantanes 2.

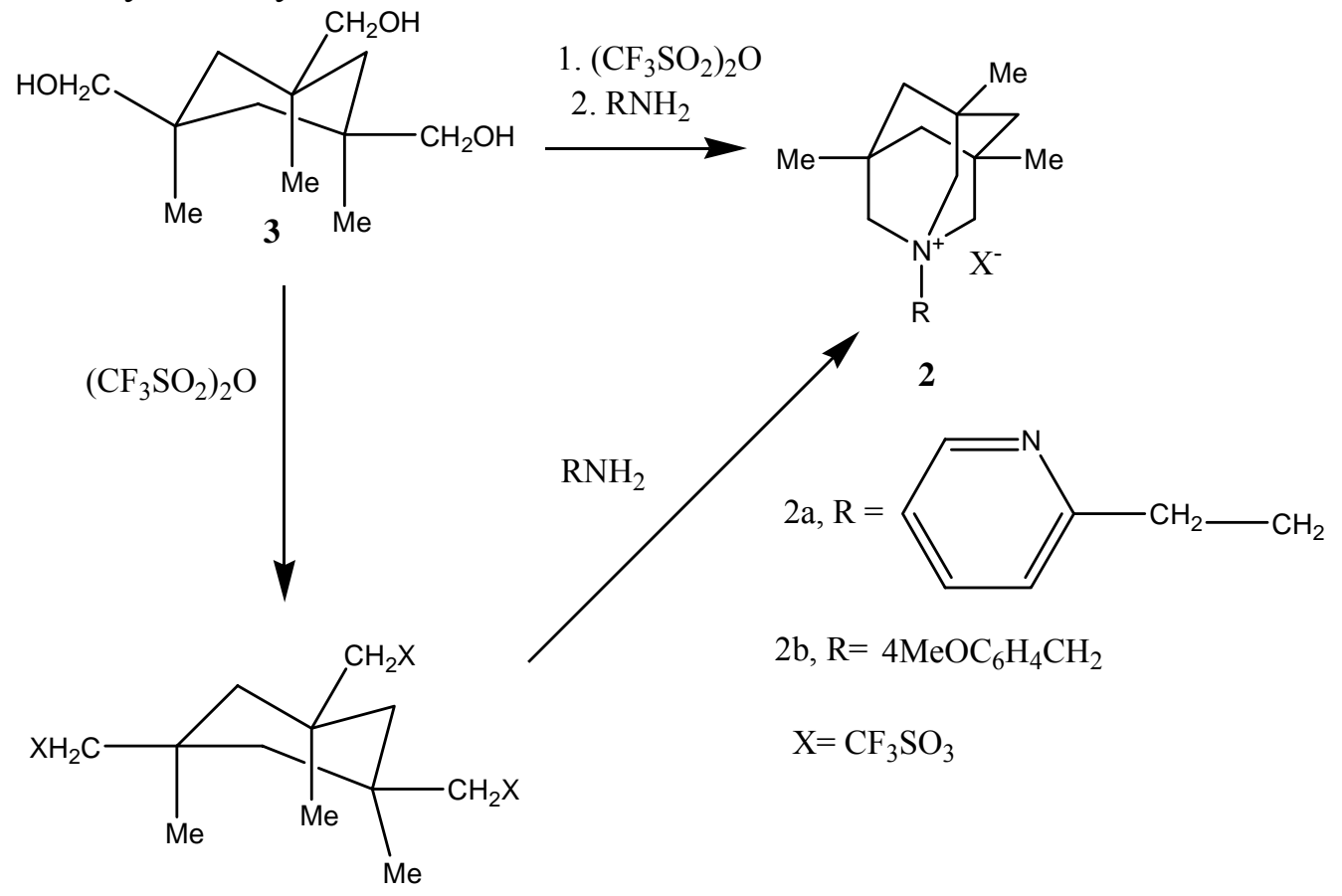

4

\section{Scheme 1}




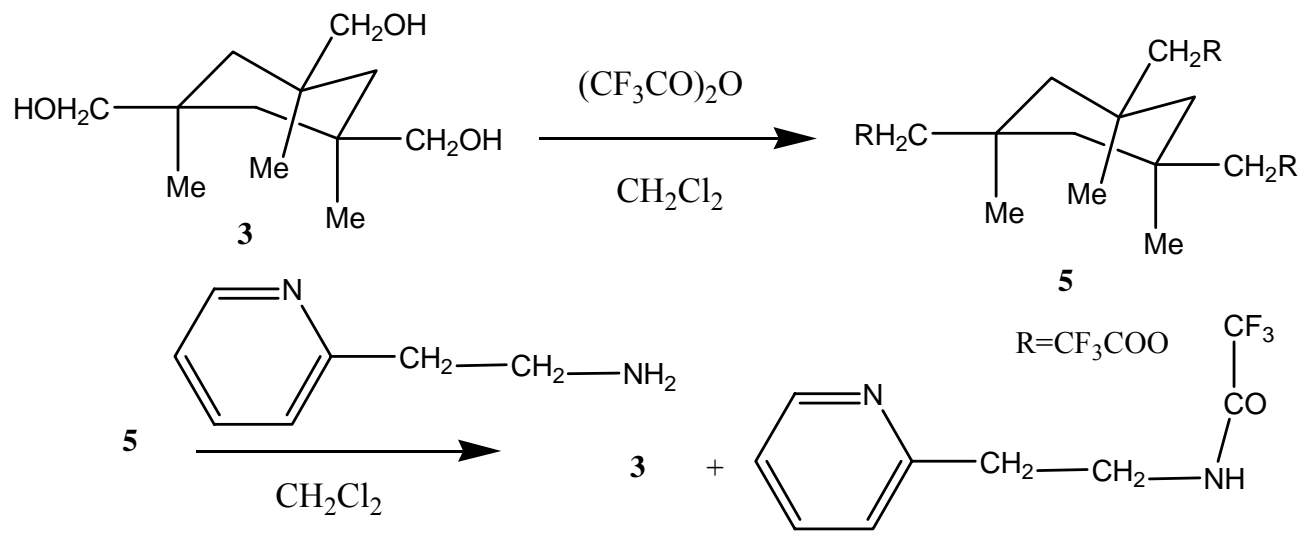

6

\section{Scheme 2}

On the other hand, the esterification of triol 3 with TFAA (4 equiv) also afforded triester 5 (Scheme 2). However, the reaction of triester 5 with 2-(2-aminoethyl)pyridine (3 equiv) did not give the corresponding azonia-adamantane, but trifluoro-acetamide 6 (Scheme 2). It is wellknown that reactions of carboxylic acid esters with amines yield acid amides. ${ }^{10}$ The low solubility of triol 3 in $\mathrm{CH} 2 \mathrm{Cl} 2$ promotes this reaction. It is suggested that trifluoroacetate lacks the function as the counter anion. The reaction of triol 3 with methanesulfonic anhydride (3 equiv) did not give the corresponding triester. The ${ }^{1} \mathrm{H}$ NMR spectrum of the reaction mixture indicated the signals of unreacted triol 3 and methanesulfonic anhydride.

Azonia-adamantane 2a was not stable in $\mathrm{CDCl} 3$. Allowing the $\mathrm{CDCl} 3$ solution of $2 \mathrm{a}$ to stand at room temperature for 5 days gave azonia-adamantane $2 \mathrm{c}(\mathrm{R}=\mathrm{H})$ and 2-vinylpyridine (Scheme $3)$. However, $2 \mathrm{a}$ did not yield $2 \mathrm{c}$ either in DMSO- $d 6$ or in acetone- $d 6$ at room temperature for 2 weeks. We have already described that acid catalysis works in chloroform. ${ }^{6,8}$ Scheme 4 shows a plausible mechanism for the reaction of $2 \mathrm{a}$ to $2 \mathrm{c}$. It has been clarified that 3,5,7-trimethyl-1-azaadamantane structures easily undergo the interconversion to the corresponding 3azabicyclo[3.3.1]nonane structures. ${ }^{1,2}$ It is suggested that azonia-adamantane $2 \mathrm{a}$ is in equilibrium with the $\mathrm{C}-\mathrm{N}$ bond cleavage form, and adhesion of a proton to the nitrogen atom in this form yields $2 \mathrm{c}$ and 2-vinylpyridine. This reaction resembles acid-catalyzed alkene formation reactions of alcohols. Azonia-adamantane 2 a reacts not only with acids but also with bases. The reaction of $2 \mathrm{a}$ with lithium methoxide in methanol afforded aza-adamantane $1^{5}$ and 2-vinylpyridine (Scheme 3). This reaction is also available for the convenient synthesis of aza-adamantane 1. Triethylamine did not react with $2 \mathrm{a}$ at all. The acidity of $\beta$-methylene protons in azoniaadamantane $2 \mathrm{a}$ has a large influence on these reactions. 

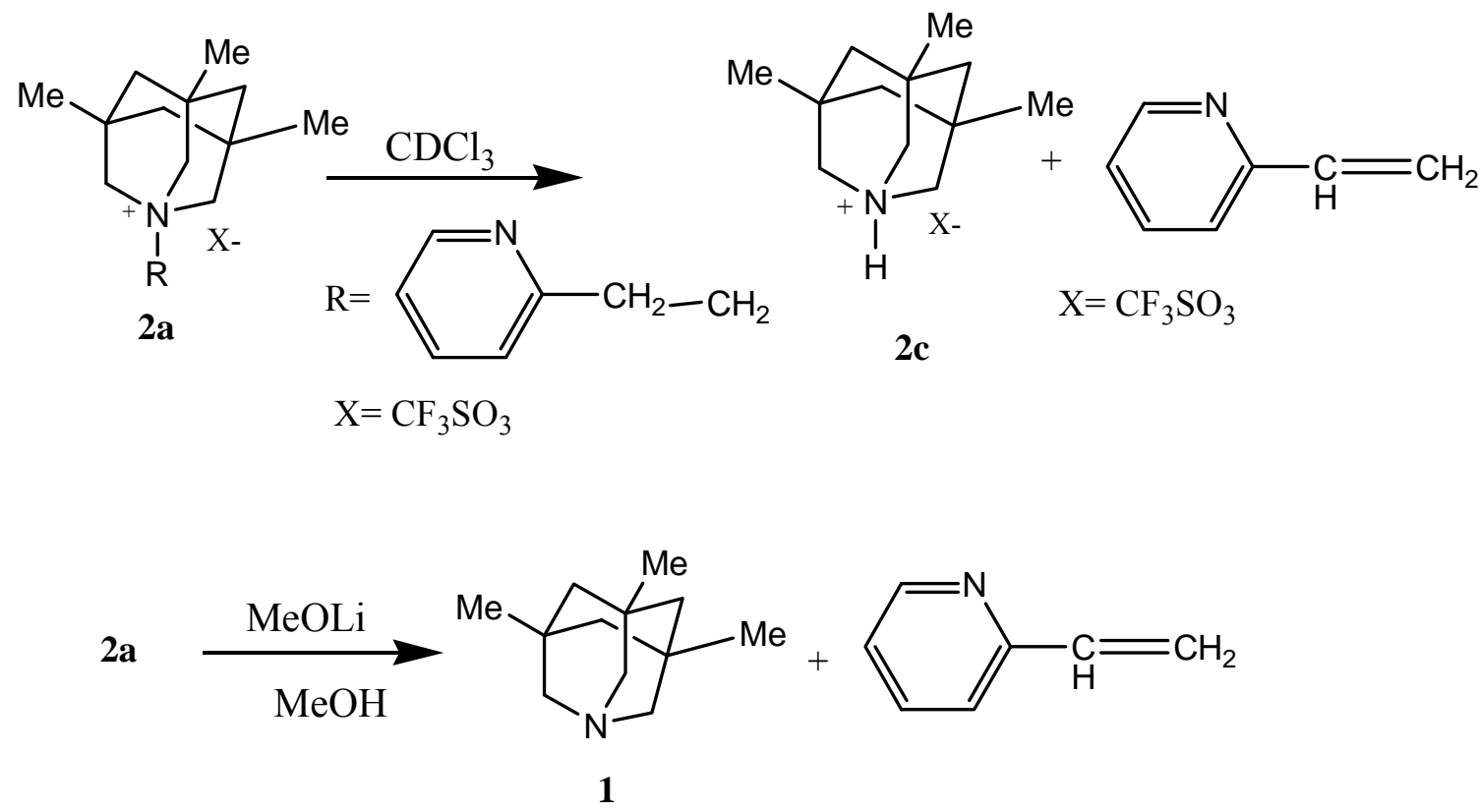

\section{Scheme 3}

\section{Conclusions}

We have described the convenient synthesis of 3,5,7-trimethyl-1-azonia-adamantanes 2 . In this synthesis, trifluoromethanesulfonate works as the key counter anion. The similar reaction of triester 5 with 2-(2-aminoethyl) pyridine does not give the corresponding azonia-adamantane, but trifluoro-acetamide 6 . We have also shown that the elimination reaction of azonia-adamantane 2 containing $\beta$-methylene protons is available for the synthesis of aza-adamantane 1 . 


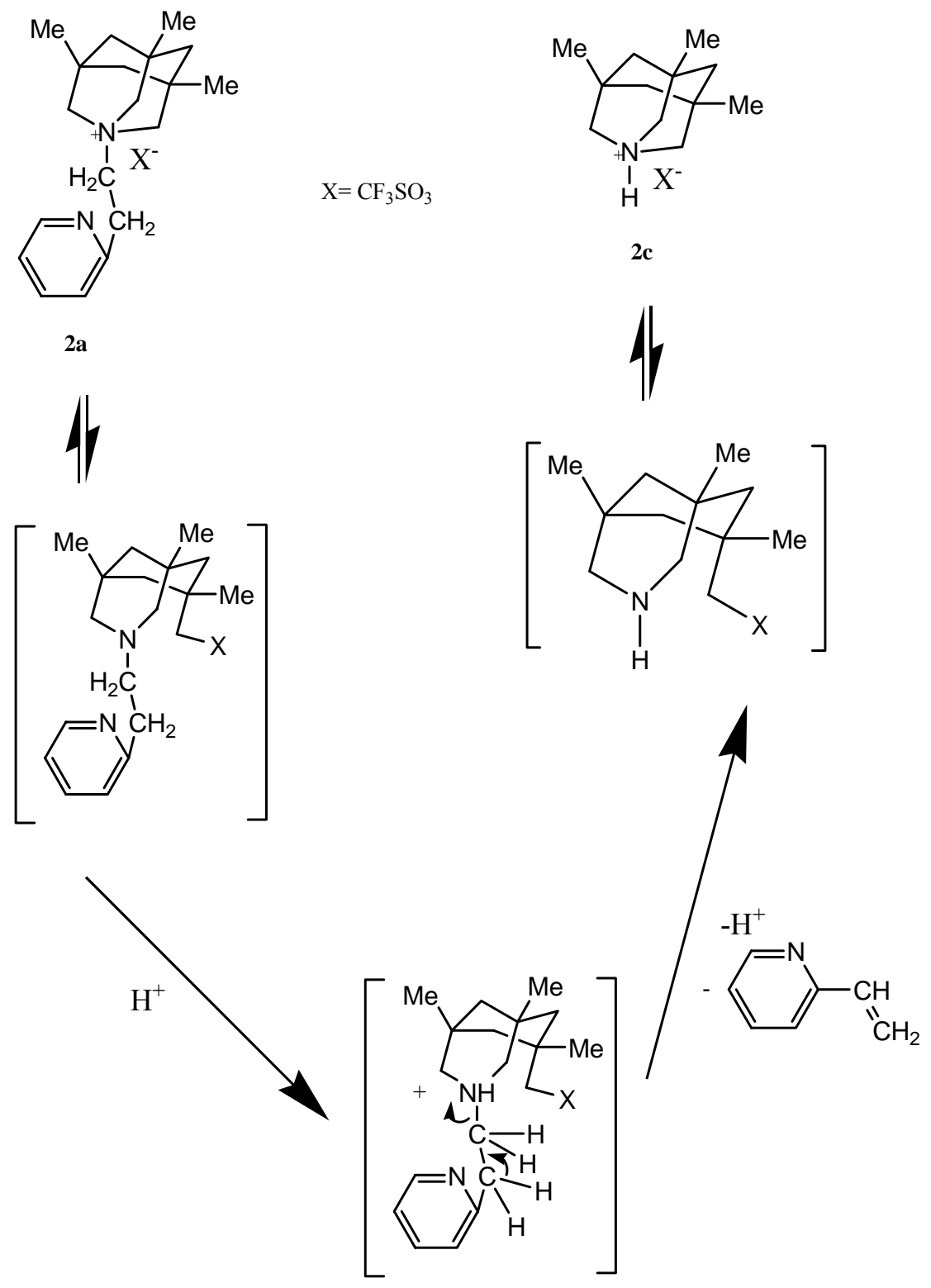

Scheme 4

\section{Experimental Section}

General Procedures. All reactions were performed in oven-dried glassware equipped with a magnetic stirring bar under an argon atmosphere, using standard syringe techniques. $\mathrm{CH}_{2} \mathrm{Cl}_{2}$ was distilled from $\mathrm{CaH}_{2}$ and stored over molecular sieves. All other solvents were of anhydrous grade. cis,cis-1,3,5-Tris(hydroxymethyl)-1,3,5-trimethylcyclohexane (3) ${ }^{6}$ and cis,cis-1,3,5trimethyl-1,3,5-tris[(trifluoromethylsulfonyloxy)methyl]cyclohexane $(4)^{7}$ were prepared by the similar procedures previously reported. All other reagents were of commercial grade. ${ }^{1} \mathrm{H}(500$ $\mathrm{MHz})$ and ${ }^{13} \mathrm{C}(125.7 \mathrm{MHz}) \mathrm{NMR}$ spectra were recorded in $\mathrm{CDCl}_{3}$, DMSO-d6, or acetone-d6. 
3,5,7-Trimethyl-1-[2-(pyridin-2-yl)ethyl]-1-azonia-adamantane trifluoromethanesulfonate (2a). A solution of trifluoromethanesulfonic anhydride $(2.2 \mathrm{~mL}, 13 \mathrm{mmol})$ in dry $\mathrm{CH}_{2} \mathrm{Cl}_{2}(15 \mathrm{~mL})$ was added to a suspension of triol $3(723 \mathrm{mg}, 3.34 \mathrm{mmol})$ and pyridine $(1.2 \mathrm{~mL})$ in $\operatorname{dry} \mathrm{CH}_{2} \mathrm{Cl}_{2}$ $(15 \mathrm{~mL})$ at $0{ }^{\circ} \mathrm{C}$. After the mixture was stirred for $2 \mathrm{~h}$ at $0{ }^{\circ} \mathrm{C}$, the precipitates were filtered, and the solution was passed through a silica gel column. Removal of the solvent under reduced pressure gave crude triester 4 . Unpurified triester 4 was dissolved in dry $\mathrm{CH}_{2} \mathrm{Cl}_{2}(10 \mathrm{~mL})$, and 2(2-aminoethyl) pyridine $(1.85 \mathrm{~g}, 15.1 \mathrm{mmol})$ was added to the solution. The solution was stirred for 3 days at room temperature, and dilute aqueous $\mathrm{NaOH}$ was added. The $\mathrm{CH}_{2} \mathrm{Cl}_{2}$ layer was separated, and the aqueous phase was extracted with $\mathrm{CH}_{2} \mathrm{Cl}_{2}$. The combined $\mathrm{CH}_{2} \mathrm{Cl}_{2}$ solutions were dried with $\mathrm{MgSO} 4$, followed by filtration, the filtrate was concentrated to remove volatiles. Azonia-adamantane $2 \mathrm{a}(338 \mathrm{mg}, 23 \%)$ was purified by recrystallization from $\mathrm{CH} 2 \mathrm{Cl} 2 /$ diethyl ether. 2a: colorless crystals; ${ }^{1} \mathrm{H}$ NMR $\left(\mathrm{CDCl}_{3}, 500 \mathrm{MHz}\right) \delta 0.99\left(9 \mathrm{H}, \mathrm{s}, \mathrm{CH}_{3}\right), 1.33\left(3 \mathrm{H}, \mathrm{d},{ }^{2} \mathrm{JHH}\right.$ $=13.0 \mathrm{~Hz}, \mathrm{CHaHe}), 1.50\left(3 \mathrm{H}, \mathrm{d},{ }^{2} \mathrm{JHH}=13.0 \mathrm{~Hz}, \mathrm{CHaHe}\right), 3.24\left(6 \mathrm{H}, \mathrm{s}, \mathrm{CH}_{2} \mathrm{~N}\right), 3.53-3.57(2 \mathrm{H}$, m, CH2CH2pyr), 3.90-3.94 (2H, m, $\left.\mathrm{CH}_{2} \mathrm{CH}_{2} \mathrm{~N}\right), 7.40-7.43(1 \mathrm{H}, \mathrm{m}, \mathrm{pyrH}), 7.85-7.87(1 \mathrm{H}, \mathrm{m}$, pyrH), 7.94-7.97 (1H, m, pyrH), 8.49-8.50 (1H, m, pyrH); ${ }^{13} \mathrm{C} \mathrm{NMR}\left(\mathrm{CDCl}_{3}, 125.7 \mathrm{MHz}\right) \delta$ $25.53\left(\mathrm{CH}_{3}\right), 28.31\left(\mathrm{CH}_{2} \mathrm{CH}_{2}\right.$ pyr $), 31.56\left[\left(\mathrm{CH}_{2}\right)_{2} \mathrm{CCH}_{3}\right], 46.07\left(\mathrm{CCH}_{2} \mathrm{C}\right), 64.81\left(\mathrm{CH}_{2} \mathrm{CH}_{2} \mathrm{~N}\right)$, $67.27\left(\mathrm{CH}_{2} \mathrm{~N}\right), 123.75$ (pyridine carbon), 126.72 (pyridine carbon), 141.04 (pyridine carbon), 145.92 (pyridine carbon), 153.88 (pyridine carbon); IR (KBr)v/ $\mathrm{cm}^{-1} 1259,1167,1026,637$; Anal. Calcd for $\mathrm{C}_{20} \mathrm{H}_{29} \mathrm{~F}_{3} \mathrm{~N}_{2} \mathrm{O}_{3} \mathrm{~S}$ : C, 55.28; H, 6.73; N, 6.45. Found: C, 55.22; H, 6.83; N, 6.51.

1-(4-Methoxybenzyl)-3,5,7-trimethyl-1-azonia-adamantane trifluoromethanesulfonate (2b). The similar manner that was employed in the preparation of $2 \mathrm{a}$ was used with triol 3 (746 mg, $3.45 \mathrm{mmol})$, trifluoromethanesulfonic anhydride $(2.0 \mathrm{~mL}, 12 \mathrm{mmol})$, and 4-methoxybenzylamine (1.91 g, $13.9 \mathrm{mmol})$. Azonia-adamantane $2 \mathrm{~b}(479 \mathrm{mg}, 1.06 \mathrm{mmol})$ was obtained in $31 \%$ yield. 2b: colorless solids; ${ }^{1} \mathrm{H} \mathrm{NMR}\left(\mathrm{CDCl}_{3}, 500 \mathrm{MHz}\right) \delta 0.91\left(9 \mathrm{H}, \mathrm{s}, \mathrm{CH}_{3}\right), 1.22\left(3 \mathrm{H}, \mathrm{d},{ }^{2} \mathrm{JHH}=\right.$ $12.8 \mathrm{~Hz}, \mathrm{CHaHe}), 1.38\left(3 \mathrm{H}, \mathrm{d},{ }^{2} \mathrm{JHH}=12.8 \mathrm{~Hz}, \mathrm{CHaHe}\right), 3.06\left(6 \mathrm{H}, \mathrm{s}, \mathrm{CH}_{2} \mathrm{~N}\right), 3.79(3 \mathrm{H}, \mathrm{s}$, $\left.\mathrm{OCH}_{3}\right), 4.49\left(2 \mathrm{H}, \mathrm{s}, \mathrm{NCH}_{2} \mathrm{Ar}\right), 6.87\left(2 \mathrm{H}, \mathrm{d},{ }^{3} \mathrm{JHH}=8.7 \mathrm{~Hz}, \mathrm{ArH}\right), 7.39\left(2 \mathrm{H}, \mathrm{d},{ }^{3} \mathrm{JHH}=8.7 \mathrm{~Hz}\right.$, $\mathrm{ArH}) ;{ }^{13} \mathrm{C} \mathrm{NMR}\left(\mathrm{CDCl}_{3}, 125.7 \mathrm{MHz}\right) \delta 25.57\left(\mathrm{CH}_{3}\right), 31.36\left[\left(\mathrm{CH}_{2}\right) 2 \mathrm{CCH}_{3}\right], 45.98(\mathrm{CCH} 2 \mathrm{C})$, $55.33(\mathrm{OCH} 3), 65.67\left(\mathrm{CH}_{2} \mathrm{~N}\right), 69.10\left(\mathrm{NCH}_{2} \mathrm{Ar}\right), 114.45$ (aromatic carbon), 117.80 (aromatic carbon), 120.75 (q, ${ }^{1} \mathrm{JCF}=320 \mathrm{~Hz}, \mathrm{CF} 3$ ), 134.67 (aromatic carbon), 161.20 (aromatic carbon); IR (KBr) $v / \mathrm{cm}^{-1} 1265,1150,1030,637$; Anal. Calcd for C21H30F3NO4S: C, 56.11; H, 6.73; N, 3.12. Found: C, 56.03; H, 6.88; N, 3.19.

cis,cis-1,3,5-Tris[(trifluoroacetoxy)methyl]-1,3,5-trimethylcyclohexane (5). A solution of trifluoroacetic anhydride $(2.0 \mathrm{~mL}, 14 \mathrm{mmol})$ in dry $\mathrm{CH}_{2} \mathrm{Cl}_{2}(15 \mathrm{~mL})$ was added to a suspension of triol $3(725 \mathrm{mg}, 3.35 \mathrm{mmol})$ and pyridine $(1.2 \mathrm{~mL})$ in dry $\mathrm{CH}_{2} \mathrm{Cl}_{2}(15 \mathrm{~mL})$ at $0{ }^{\circ} \mathrm{C}$. After the mixture was stirred for $2.5 \mathrm{~h}$ at $0^{\circ} \mathrm{C}$, the solution was passed through a silica gel column. Removal of the solvent under reduced pressure gave triester 5 (1.59 g, 94\%). 5: colorless solids; ${ }^{1} \mathrm{H} \mathrm{NMR}\left(\mathrm{CDCl}_{3}, 500 \mathrm{MHz}\right) \delta 1.25\left(9 \mathrm{H}, \mathrm{s}, \mathrm{CH}_{3}\right), 1.32\left(3 \mathrm{H}, \mathrm{d}, \mathrm{AB},{ }^{2} \mathrm{JHH}=14.2 \mathrm{~Hz}, \mathrm{CHaHe}\right)$, $1.34\left(3 \mathrm{H}, \mathrm{d}, \mathrm{AB},{ }^{2} \mathrm{JHH}=14.2 \mathrm{~Hz}, \mathrm{CHaHe}\right), 3.94(6 \mathrm{H}, \mathrm{s}, \mathrm{CH} 2 \mathrm{O}) ;{ }^{13} \mathrm{C} \mathrm{NMR}\left(\mathrm{CDCl}_{3}\right.$, $125.7 \mathrm{MHz}) \delta 25.43\left(\mathrm{CH}_{3}\right), 34.27\left[\left(\mathrm{CH}_{2}\right)_{2} \mathrm{CCH}_{3}\right], 38.85\left(\mathrm{CCH}_{2} \mathrm{C}\right), 77.40\left(\mathrm{CH}_{2} \mathrm{O}\right), 114.48\left(\mathrm{q},{ }^{1} \mathrm{JCF}\right.$ 
$\left.=285 \mathrm{~Hz}, \mathrm{CF}_{3}\right), 157.38\left(\mathrm{q},{ }^{2} \mathrm{JCF}=43 \mathrm{~Hz}, \mathrm{COO}\right)$; Anal. Calcd for $\mathrm{C}_{18} \mathrm{H}_{21} \mathrm{~F}_{9} \mathrm{O}_{6}: \mathrm{C}, 42.87 ; \mathrm{H}, 4.20$. Found: C, 43.54; H, 4.14 .

2,2,2-Trifluoro- $N$-[2-(pyridin-2-yl)ethyl]-acetamide (6). 2-(2-aminoethyl)pyridine (1.28 g, $10.4 \mathrm{mmol})$ was added to a $\mathrm{CH}_{2} \mathrm{Cl}_{2}$ solution $(10 \mathrm{~mL})$ of triester $5(1.59 \mathrm{~g}, 3.16 \mathrm{mmol})$. While the solution was stirred for $3 \mathrm{~h}$ at room temperature, precipitates were formed. The precipitates were filtered and washed with diethyl ether. Triol $3(658 \mathrm{mg}, 3.04 \mathrm{mmol})$ was obtained in 96\% yield. After the filtrate was concentrated to remove volatiles, the residue was extracted with $\mathrm{CH}_{2} \mathrm{Cl}_{2}$. Removal of the solvent under reduced pressure gave acetamide 6 (1.99 g, 96\%). Further purification was carried out by recrystallization from diethyl ether/hexane. 6: pale yellow crystals; ${ }^{1} \mathrm{H}$ NMR $\left(\mathrm{CDCl}_{3}, 500 \mathrm{MHz}\right) \delta 3.00-3.02\left(2 \mathrm{H}, \mathrm{m}, \mathrm{CH}_{2}\right), 3.72-3.75\left(2 \mathrm{H}, \mathrm{m}, \mathrm{CH}_{2}\right), 7.13-$ 7.16 (2H, m, pyrH), 7.59-7.63 (1H, m, pyrH), 8.40 (1H, br. s, NH), 8.47-8.48 (1H, m, pyrH); ${ }^{13} \mathrm{C}$ NMR $\left(\mathrm{CDCl}_{3}, 125.7 \mathrm{MHz}\right) \delta 35.13\left(\mathrm{CH}_{2}\right), 38.66\left(\mathrm{CH}_{2}\right), 115.97\left(\mathrm{q},{ }^{1} J C F=287 \mathrm{~Hz}, \mathrm{CF}_{3}\right)$, 121.90 (pyridine carbon), 123.43 (pyridine carbon), 136.91 (pyridine carbon), 149.02 (pyridine carbon), 156.97 (q, ${ }^{2} \mathrm{JCF}=37 \mathrm{~Hz}, \mathrm{CO}$ ), 158.94 (pyridine carbon); IR (KBr)v/ cm ${ }^{-1} 3202,1724$; Anal. Calcd for $\mathrm{C}_{9} \mathrm{H}_{9} \mathrm{~F}_{3} \mathrm{~N}_{2} \mathrm{O}$ : C, 49.55; H, 4.16; N, 12.84. Found: C, 49.62; H, 4.19; N, 12.81 .

3,5,7-Trimethyl-1-aza-adamantane (1). ${ }^{5}$ A solution of azonia-adamantane $2 \mathrm{a}$ (76.3 $\mathrm{mg}$, $0.18 \mathrm{mmol})$ in methanol $(20 \mathrm{~mL})$ was added to a $12 \%$ lithium methoxide methanol solution $(2 \mathrm{~mL})$ at $0{ }^{\circ} \mathrm{C}$. After the mixture was stirred for $18 \mathrm{~h}$ at room temperature, water $(1 \mathrm{~mL})$ was added to the solution. Volatiles were removed under reduced pressure, and the residue was extracted with $\mathrm{CH}_{2} \mathrm{Cl}_{2}$. Removal of the solvent under reduced pressure gave aza-adamantane $1^{5}$ (24.9 mg, 79\%).

NMR Monitoring experiment. Azonia-adamantane $2 \mathrm{a}(2.1 \mathrm{mg}, 0.005 \mathrm{mmol})$ was added to $\mathrm{CDCl}_{3}(0.8 \mathrm{~mL})$ in an NMR tube. Allowing the solution to stand at room temperature for 5 days gave azonia-adamantane $2 \mathrm{c}(\mathrm{R}=\mathrm{H})$ and 2-vinylpyridine. $2 \mathrm{c}:{ }^{1} \mathrm{H} \mathrm{NMR}\left(\mathrm{CDCl}_{3}, 500 \mathrm{MHz}\right) \delta 0.96$ $\left(9 \mathrm{H}, \mathrm{s}, \mathrm{CH}_{3}\right), 1.37\left(3 \mathrm{H}, \mathrm{d},{ }^{2} \mathrm{JHH}=12.7 \mathrm{~Hz}, \mathrm{CHaHe}\right), 1.44\left(3 \mathrm{H}, \mathrm{d},{ }^{2} \mathrm{JHH}=12.7 \mathrm{~Hz}, \mathrm{CHaHe}\right), 3.00$ $\left(6 \mathrm{H}, \mathrm{s}, \mathrm{CH}_{2} \mathrm{~N}\right), 10.70\left(1 \mathrm{H}\right.$, br. s, NH); ${ }^{13} \mathrm{C} \mathrm{NMR}\left(\mathrm{CDCl}_{3}, 125.7 \mathrm{MHz}\right) \delta 25.89\left(\mathrm{CH}_{3}\right), 30.05$ $\left[\left(\mathrm{CH}_{2}\right)_{2} \mathrm{CCH}_{3}\right], 47.22\left(\mathrm{CCH}_{2} \mathrm{C}\right), 59.53\left(\mathrm{CH}_{2} \mathrm{~N}\right)$.

\section{References}

1. (a) Kirby, A. J.; Komarov, I. V.; Feeder, N. J. Am. Chem. Soc. 1998, 120, 7101. (b) Kirby, A. J.; Komarov, I. V.; Wothers, P. D.; Feeder, N. Angew. Chem., Int. Ed. 1998, 37, 785.

2. Risch, N.; Langhals, M.; Mikosch, W.; Bögge, H.; Müller, A. J. Am. Chem. Soc. 1991, 113, 9411.

3. Udding, J. H.; Papin, N.; Hiemstra, H.; Speckamp, W. N. Tetrahedron 1994, 50, 8853.

4. (a) Beecham Group PLC Chem. Abstr. 1988, 108, 5870s. (b) Jarreau, F. X.; Koenig, J. J. Chem. Abstr. 1985, 102, 131937h. (c) Jarreau, F. X.; Koenig, J. J. Chem. Abstr. 1983, 99, $88055 \mathrm{z}$. 
5. (a) Risch, N.; Billerbeck, U.; Meyer-Roscher, B. Chem. Ber. 1993, 126, 1137. (b) Risch, N.; Billerbeck, U.; Krieger, E. Chem. Ber. 1992, 125, 459.

6. Izumi, H.; Setokuchi, O.; Shimizu, Y.; Tobita, H.; Ogino, H. J. Org. Chem. 1997, 62, 1173.

7. Mayer, H. A.; Fawzi, R.; Steimann, M. Chem. Ber. 1993, 126, 1341.

8. Izumi, H.; Futamura, S. J. Org. Chem. 1999, 64, 4502.

9. Izumi, H.; Futamura, S. J. Chem. Soc., Perkin Trans. 1 1998, 1925.

10. Allen, C. F. H.; Humphlett, W. J. Org. Synth. 1963, 4, 80. 